\title{
Punctual Breast Implant Rupture following Lipofilling: Only a Myth?
}

\author{
Andres Rivera Carlota González-Pozega Gorka Ibarra \\ Borja Fernandez-lbarburu Ángela García-Ruano Alfonso Vallejo-Valero
}

Plastic Surgery Department, Gregorio Marañon General University Hospital, Madrid, Spain

\section{Established Facts}

- Unilateral capsular contracture could be a symptom of an early implant rupture.

- Ultrasound is the first exploration recommended to evaluate breast implant complications.

- Treatment of capsular contracture is surgery with implant removal and capsulotomy or capsulectomy.

\section{Novel Insights}

- Ultrasound can misdiagnose punctual breast implant ruptures.

- Lipofilling in a prosthetic breast can lead to a fat deposit inside the implant.

- In the case of intraprosthetic lipofilling, the indication of reintervention is based on symptoms as surgery confirms the diagnosis and resolves the clinical chart.

\section{Keywords}

Breast cancer · Surgical therapy · Ultrasound

\section{Abstract}

Background: Lipofilling techniques are widespread in clinical practice as a complement to breast reconstruction, despite posing some risk. Punctual implant rupture following a fat transfer is one of the possible complications, which has not been properly reported yet and is probably being underdiagnosed. The aim of this paper is to report key facts for appropriate diagnosis of this clinical chart. Case Report: We present the case of a 47-year-old woman with a bilateral prosthetic breast reconstruction who was treated with autologous fat graft for upper pole enhancing and scar improvement. The patient developed an early unilateral breast capsular contracture after the fat graft procedure, with normal radiological exploration. Surgical findings showed intra- prosthetic fat deposits and a punctual implant rupture. Conclusions: Punctual breast implant rupture is a possible complication of lipofilling that is usually not suspected at first consultation and might be underdiagnosed based on radiological findings, so investigating clinical signs should necessarily be a prerequisite to diagnosis.

(c) 2020 S. Karger AG, Basel

\section{Introduction}

Lipofilling is a technique that consists in the transfer of autologous fat, after processing, from a fat deposit of the patient (i.e., lower abdomen) to another site, where it is supposed to serve a particular function. The procedure is widely used in breast reconstruction with multiple indications that range from the treatment of lumpectomy defects [1] to the remodeling of prosthetic breasts (i.e., 
Fig. 1. Preoperative patient status: asymmetrical capsular contracture can be appreciated, with double bubble deformity on the right breast.

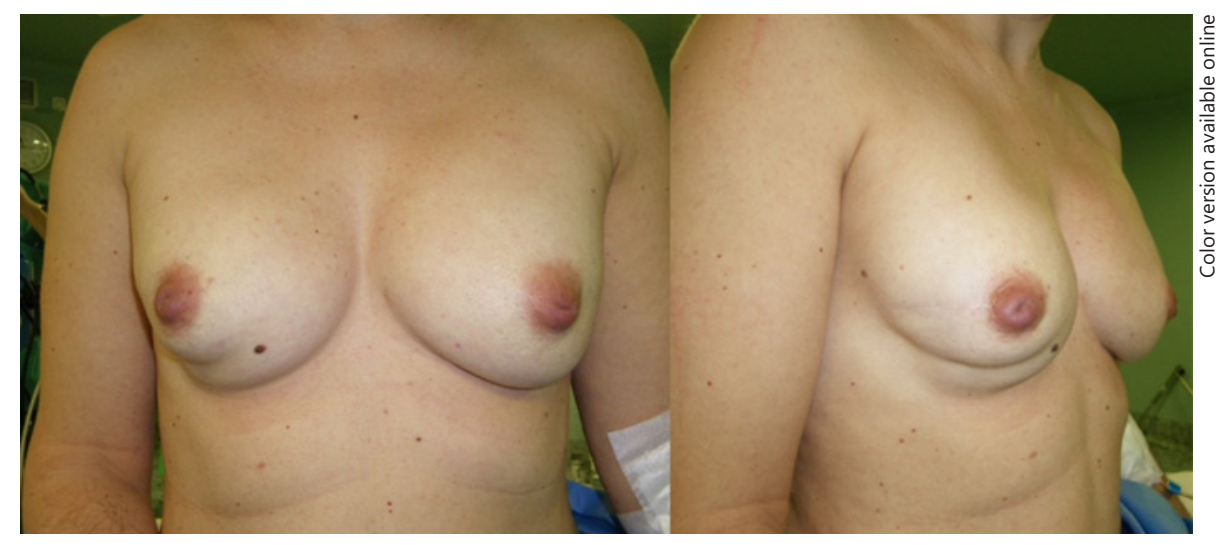

Fig. 2. Macroscopic implant detail. Fatty locules are shown inside the capsular implant.

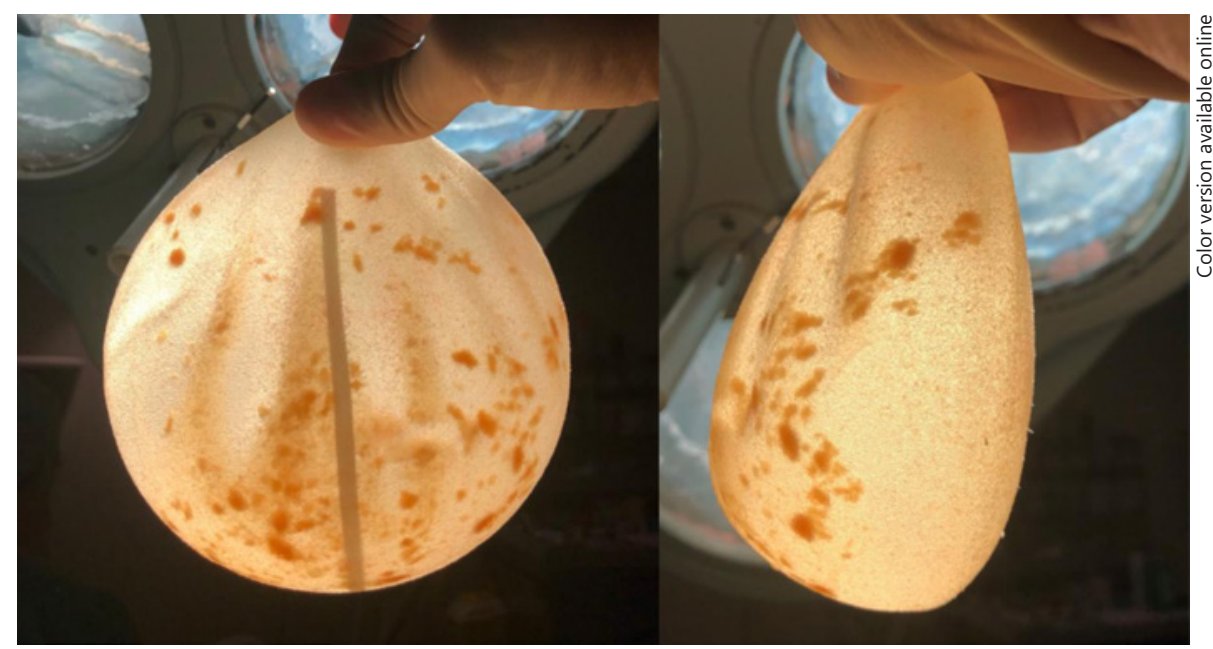

low pole enhancing) or autologous flaps (i.e., latissimus dorsi reconstruction) in radical mastectomies. Several studies suggest that fat transfer is a safe procedure at an oncological level [1-4] as well as a useful tool for correction of focal defects [5] and treating postoperative pain [6].

The risks of lipofilling have been documented with an incidence that varies from 0 to $9 \%[4,7]$. Despite this fact, some unusual complications have not yet been properly reported, such as those associated with procedures performed on breast implants, one of which is implant rupture.

Here, we present a clinical case of punctual breast implant rupture following fat transfer. Literature of lipofilling complications is reviewed, and diagnostic tips for appropriate assessment of this complication are described.

\section{Case Report and Case Presentation}

A 47-year-old woman with high genetic breast carcinoma risk, underwent bilateral subcutaneous mastectomy with areola conservation and submuscular bilateral reconstruction with CPG - 350-
mL silicone gel prostheses, CPG Mentor ${ }^{\circledR}$ (Johnson \& Johnson Medical Devices \& Diagnostic Group - Latin America L.L.C.).

One year later, a lipotransfer was performed to treat irregularities in the skin contour in the right breast. Following Coleman's technique, $80 \mathrm{~mL}$ of processed fat proceeding from the lower abdomen was transferred to the low pole of the right breast using a 21 Gauss diameter blunt cannula.

The immediate postoperative period was uneventful, but the patient progressively developed a unilateral grade IV capsular contracture 6 months after surgery (Fig. 1). Breast ultrasound and magnetic resonance studies revealed neither signs of prosthetic rupture nor periprosthetic seroma.

Due to the persistence of the symptoms, intervention was performed for appropriate implant and capsular analysis. The right breast showed significant contracture without associated seroma. Gross examination of the implant demonstrated the presence of intraprosthetic fatty accumulations (Fig. 2) and located weaknesses at its cover without extrusion of the silicone content. These signs assured the diagnosis of punctual implant rupture.

Unilateral capsulectomy with implant replacement was performed. The implant was replaced with a new $350-\mathrm{mL}$ silicone gel anatomical implant, CPG Mentor ${ }^{\circledR}$ (Johnson \& Johnson Medical Devices \& Diagnostic Group - Latin America L.L.C.) for breast reconstruction.

No acute or late complications nor episodes of contracture have been reported after a 1-year follow-up period. Patient satisfaction was achieved with a good esthetic outcome. 


\section{Discussion and Conclusion}

The present case is considered the model for punctual implant rupture that may arise when performing a lipofilling on a prosthetic reconstructed breast. The literature review shows no reports of this complication, whose clinical presentation consists in unilateral breast capsular contracture after fat transfer without radiological findings. The possibility of implant puncture following the procedure should be addressed if an asymmetric clinic is presented.

Main complications of lipofilling on prosthetic breasts include fatty necrosis, followed by fatty cysts, cellulite, abscesses, capsular contracture, and prosthetic exposure [3, $7,8]$. Capsular contracture is involved in $17.9 \%$ of all complications [6].

On the other hand, some studies in patients with capsular contracture, even in irradiated fields, describe a therapeutic effect of the fat graft, allowing capsular contracture to be reduced [7] and the associated pain to be reduced [9]. The interaction of adipose tissue-derived stem cells with the fibroblast population is thought to be responsible for these effects [7]. The present facts contrast with the symptoms of our patient, which point to an implant rupture after the lipofilling process.

Image diagnosis of implant rupture is usually achieved by echography or MRI studies. MRI is considered gold standard for diagnosis, yielding a higher accuracy than echography with a sensitivity of $89 \%$, and a specificity of 97\% [10]. Since the clinical presentation of punctual implant rupture consists of capsular contracture with no radiological signs, it must be supposed that a considerable percentage of capsular contracture patients may have this unnoticed complication.

Presurgical definition of infiltration planes as well as prosthetic distraction maneuvers should be used to avoid this complication during the procedure [11]. Therefore, in our opinion, the benefits of lipofilling in patients with prosthetic reconstruction and a slight cutaneous tissue outweigh the risks.

Currently, ultrasound is an available tool for plane definition before infiltration. In this regard, pre- or intraop- erative ultrasound allows the surgeon to study patient tissues in a low-cost and noninvasive way, minimizing punctual implant ruptures and optimizing the results of the technique [11].

The present case demonstrates that the absence of radiological rupture signs does not preclude a punctual implant rupture diagnosis. From these results, it is clear that clinic should be considered above the rest of the data as essential in diagnosing punctual implant rupture. If unilateral early contracture is developed after breast lipofilling, a capsular perforation should be suspected.

In conclusion, this detailed description of punctual implant rupture due to a lipofilling should serve as a template to assess its real incidence, since this complication is mostly unknown and underdiagnosed.

\section{Statement of Ethics}

The patient has given her written informed consent to publish her case, including publication of images.

\section{Conflict of Interest Statement}

The authors have no conflicts of interest to declare.

\section{Funding Sources}

The authors did not receive any funding.

\section{Author Contributions}

Andres Rivera and Alfonso Vallejo-Valero performed the surgery and collected the clinical data and illustrations. Borja Fernandez-Ibarburu encouraged Andres Rivera to investigate the clinical findings and helped with the literature review. Ángela GarcíaRuano, Carlota González-Pozega, and Gorka Ibarra contributed to the writing of the manuscript. Andres Rivera and Alfonso VallejoValero supervised the final version.

\section{References}

1 Kümmel A, Kümmel S, Blohmer JU, Faridi A, Nitz U, Loibl S, et al. Autologous Lipotransfer - Daily Therapeutic Practice in Breast Cancer: An Intergroup Analysis Encompassing NOGGO, WSG, GBG, AWO Gyn and DGPRÄC. Breast Care (Basel). 2019 Jun;14(3):165-9.

2 Khan LR, Raine CR, Dixon JM. Immediate lipofilling in breast conserving surgery. Eur J Surg Oncol. 2017 Aug;43(8):1402-8.
3 Groen JW, Negenborn VL, Twisk DJ, Rizopoulos D, Ket JC, Smit JM, et al. Autologous fat grafting in onco-plastic breast reconstruction: A systematic review on oncological and radiological safety, complications, volume retention and patient/surgeon satisfaction. J Plast Reconstr Aesthet Surg. 2016 Jun;69(6):742-64.

4 Pearl RA, Leedham SJ, Pacifico MD. The safety of autologous fat transfer in breast cancer: lessons from stem cell biology. J Plast Reconstr Aesthet Surg. 2012 Mar;65(3):283-8.
5 Rietjens M, De Lorenzi F, Rossetto F, Brenelli F, Manconi A, Martella S, et al. Safety of fat grafting in secondary breast reconstruction after cancer. J Plast Reconstr Aesthet Surg. 2011 Apr;64(4):477-83.

6 Komorowska-Timek E, Turfe Z, Davis AT. Outcomes of Prosthetic Reconstruction of Irradiated and Nonirradiated Breasts with Fat Grafting. Plast Reconstr Surg. 2017 Jan; 139(1):1e-9e. 
7 Panettiere P, Marchetti L, Accorsi D. The serial free fat transfer in irradiated prosthetic breast reconstructions. Aesthetic Plast Surg. 2009 Sep;33(5):695-700.

8 Missana MC, Laurent I, Barreau L, Balleyguier C. Autologous fat transfer in reconstructive breast surgery: indications, technique and results. Eur J Surg Oncol. 2007 Aug;33(6):68590.
9 Cuomo R, Zerini I, Botteri G, Barberi L, Nisi G, D’Aniello C. Postsurgical pain related to breast implant: Reduction with lipofilling procedure. In Vivo. 2014 Sep-Oct;28(5):9936 .

10 Hölmich LR, Vejborg I, Conrad C, Sletting S, McLaughlin JK. The diagnosis of breast implant rupture: MRI findings compared with findings at explantation. Eur J Radiol. 2005 Feb;53(2):213-25.
11 Scotto di Santolo M, Sagnelli M, Tortora G, Santoro MA, Canta PL, Molea G, et al. The utility of the high-resolution ultrasound technique in the evaluation of autologous adipose tissue lipofilling, used for the correction of post-surgical, post-traumatic and post-burn scars. Radiol Med (Torino). 2016 Jun;121(6): 521-7. 